\title{
An assessment of the usefulness of dip slides in a children's hospital
}

\author{
T. L. SHRESTHA AND N. E. G. RICHARDSON \\ From Queen Mary's Hospital for Children, Carshalton, Surrey
}

SYNOPSIS The introduction of the dip inoculum technique has made possible the immediate culture of urines which in the normal way might take a number of hours to reach the laboratory. We have used this technique in conjunction with conventional methods of culture to try to ascertain whether the delay in transit normally encountered in this hospital affects materially the clinical significance of the results. We have not been able to demonstrate any advantage in the dip inoculum technique in the circumstances in which we have used it in the present study.

Since the introduction by Mackey and Sandys (1965) of the dip inoculum technique for the culture of urine, its usefulness and reliability have been assessed in various laboratories and a comparison has been made between this method and more traditional methods of culture (Guttman and Naylor, 1967). The object of this study is to see if the dip inoculum method offers any advantage over other methods in the routine laboratory of a children's hospital. It has been stated that delay in the despatch of specimens from the wards may give rise to erroneous results and it would seem therefore that immediate culture by the dip slide technique might enable the differentiation between true infection and contamination to be made more easily. Comparison of the growth on the dip slide with the growth on conventional culture plates should demonstrate whether or not the delay in transit affects the number of colonies grown.

\section{Methods}

The dip slide kit used was that manufactured by Oxoid. One side of the slide is coated with McConkey's medium and the other with CLED medium.

The method of urine collection in this hospital is by plastic bag in children under 2 years of age. The child passes urine into the bag and this is subsequently released into a sterile universal container through a tube in the side of the bag. Recently a modified collecting bag, the U-bag manufactured by Hollister of the USA, has been in use and has proved successful. This consists of a second

Received for publication 31 July 1973. bag into which urine passes through a small aperture. This precludes continuing contact with the tissues surrounding the urethra. In children over 2 years of age urine specimens are collected by the usual midstream technique.

It has been found that specimens collected as above may be delayed on the wards for anything up to four hours, depending on the availability of porters. In this investigation the nursing staff noted the time the specimen was collected and the laboratory staff noted its time of arrival and immediate culture in the laboratory. We therefore have a measure of the lapse of time between the voiding of the urine and the plating of the specimen onto the culture media plates. The urines were plated on blood and McConkey's solid media with a standard loop holding approximately $0.004 \mathrm{ml}$ of urine. The dip slide and culture plates were incubated at $37^{\circ} \mathrm{C}$ overnight. The following morning the bacterial growth on the McConkey medium of the dip slide was scored according to the manufacturer's instruction. The bacterial growth on the McConkey's plate was scored by the same person as 'heavy', 'moderate', or 'scanty'. The heavy growth was equivalent to 100000 or more colonies per $\mathrm{ml}$ of urine, moderate corresponded to $10000-100000$ colonies per $\mathrm{ml}$, and scanty to less than 10000 per $\mathrm{ml}$. Those dip slides showing fewer than 10000 colonies per $\mathrm{ml}$ and those showing scanty growth were designated 'no significant bacterial growth', those with 10000-100 000 colonies per $\mathrm{ml}$ on the plates and dip slides were classified as doubtful cases, and those showing more than 100000 colonies per $\mathrm{ml}$ by either method were assumed to be true urinary tract infections. 


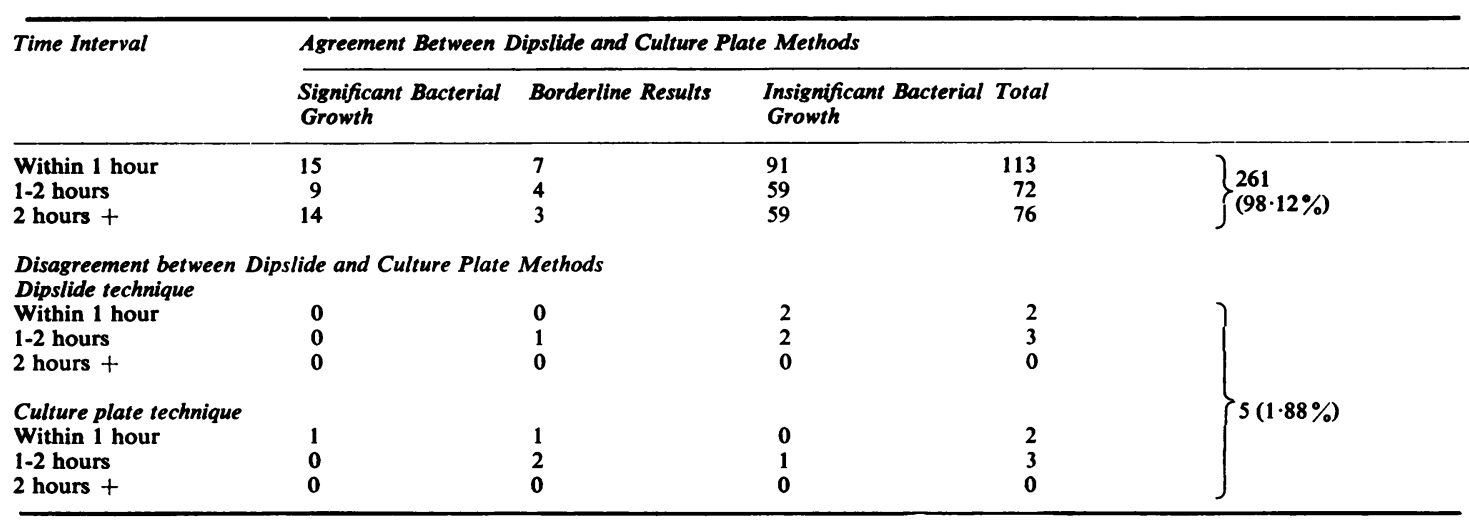

Table

\section{Results}

In order to find out the significance of the delay in transit time, we grouped the results under three headings: (1) those reaching the laboratory within an hour of collection; (2) those with one to two hours' delay; and those with more than two hours' delay. The total number of specimens was 266 and of that number 261 gave similar results by both methods of culture $(98 \cdot 12 \%)$. There was disagreement in five cases $(1.88 \%)$. Of these five cases, 4 specimens grew less than 10000 colonies per $\mathrm{ml}$ on dip slides, while three of the culture plates grew between 10000 and 100000 colonies per $\mathrm{ml}$, and one plate had 100000 colonies per $\mathrm{ml}$ of urine. One culture plate had insignificant growth but the dip slide had 10 000-100 000 colonies per $\mathrm{ml}$ (see table). About $29 \%$ of the specimens showing agreement by the two methods reached our laboratory after two or more hours' delay. The five cases which disagreed with each other in their bacterial counts reached the laboratory within two hours of collection.

\section{Conclusion}

The results we have obtained clearly indicate that the measure of agreement between the dip slide and culture plate techniques is very close. In our hospital practice the dip slide method appears to offer no advantage over the standard loop method of plating the urine on culture plates (Guttman and Stokes, 1963). The use of dip slides by general practitioners offers obvious advantages which have been well documented. Recently Jackaman, Darrell, and Shackman (1973) have demonstrated the usefulness of the dip slide technique as a screening procedure in a urological unit, but we would agree with them that subsequent definitive bacteriology may involve ate increase rather than a decrease in the laboratory work load. It is interesting that $29 \%$ of the urine specio mens which agreed by both techniques reached the laboratory after two or more hours' delay in transit. These specimens had not been stored in refrigerators before being sent to the laboratory. We plan to make a further study of the effect of delayed culture on the number of organisms grown. In this hospital at least delays of up to four hours do not appear to influence the clinical significance of the result.

We would like to thank the staff of the Pathology Department and the nursing staff of this hospital for their help in this investigation.

\section{References}

Guttman, D., and Naylor, G. R. E. (1967). Dip-slide: an aid to quantitative urine culture in general practice. Brit. med. J., 3, 343-345.

Guttman, D., and Stokes, E. J. (1963). Diagnosis of urinary infection. Brit. med. J., 9, 1384-1387.

Mackey, J. P., and Sandys, G. H. (1965). Laboratory diagnosis of the urinary tract in general practice by means of a dipinoculum transport medium. Brit. med. J., 2, 1286-1288.

Jackaman, Darrell, J. H., and Shackman R. (1973). The dip-slide in urology. Brit. med. J., 1, 207-208. 